\title{
Detection of laser induced dielectric breakdown in water using a laser doppler vibrometer
}

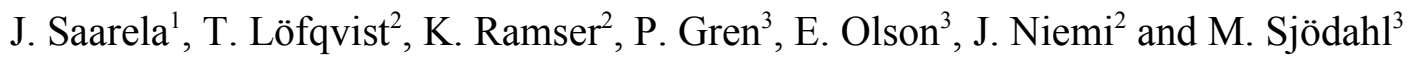 \\ ${ }^{1}$ Department of Electrical and Information Engineering and Infotech Oulu, University of Oulu, P.O. \\ Box 4500, 90014 University of Oulu, Finland, fax +358-8-553 2774, juha.saarela@oulu.fi \\ ${ }^{2}$ Department of Computer Science and Electrical Engineering, Luleå University of Technology, SE- \\ 97187 Luleå, Sweden \\ ${ }^{3}$ Department of Applied Physics and Mechanical Engineering, Luleå University of Technology, SE- \\ 97187 Luleå, Sweden
}

\begin{abstract}
This study is focused on exploring the feasibility of an all-optic surface scanning method in determining the size and position of a submerged, laser generated, optoacoustic source. The optoacoustic effect was here generated when the absorption of a short electromagnetic pulse in matter caused a dielectric breakdown, a plasma emission flash and a subsequent acoustic wave. In the experiment, a laser pulse with $\lambda=1064 \mathrm{~nm}$ and $12 \mathrm{~ns}$ pulse length was aimed at a volume of deionized water. When the laser beam was focused by a $f=16 \mathrm{~mm}$ lens, a single dielectric breakdown spot occurred. When a $f=40 \mathrm{~mm}$ was used several breakdowns in a row were induced. The breakdowns were photographed using a double shutter camera. The acoustic wave generated by the dielectric breakdowns were detected at a point on the water surface using a laser Doppler vibrometer (LDV). First, the LDV signal was used to calculate the speed of sound with an accuracy of $10 \mathrm{~m} / \mathrm{s}$. Secondly, the location and length of the dielectric breakdown was calculated with an accuracy of $1 \mathrm{~mm}$. The calculated position matched the breakdown location recorded by a camera. The results show that it is possible to use LDV surface measurements from a single spot to determine both the position and length of the OA source as well as the speed of sound in the medium. Furthermore, the LDV measurements also show a secondary peak that originates from the OA source. To unravel the origin and properties of this interesting feature, further investigations are necessary.
\end{abstract}

\section{PACS:}

42.30.Wb Image reconstruction; tomography

42.62.Cf Industrial applications

42.79.Qx Range finders, remote sensing devices; laser doppler velocimeters, SAR and LIDAR

43.30.+m Underwater sound

43.35. $+\mathrm{d}$ Ultrasonic, quantum acoustics and physical effects of sound 


\section{INTRODUCTION}

In laser based optoacoustics (OA) the interaction between a laser pulse and a material acts as a source of acoustic waves. There are mainly five different mechanisms that lie behind the conversion from electromagnetic to mechanical energy; dielectric breakdown, vaporization, chemical reactions, thermoelastic process and electrostriction. The strength of the OA source is in a falling scale from dielectric breakdown being the strongest to electrostriction which is the weakest. The basic theory of laser-induced breakdown in aqueous media has been summarized by Kennedy et al. [1].

The OA effect is utilized in various fields and most recently also in laser medicine where the resulting pressure wave can be used to create wanted effects, like in angioplasty for dilation of blood vessels [2] or to use the understanding of $\mathrm{OA}$ in avoiding unwanted effects as in laser based eye microsurgery [3] where sensitive tissue should not be damaged by the pressure wave.

Laser based OA has a high potential for imaging purposes within biomedical applications. An OA imaging system comprises a pulsed laser, a suitable detection system and evaluation tools in order to analyze the surface wave as well as the OA source. One advantage of OA imaging compared to other methods is that it combines the high contrast provided by optical imaging with the high ultrasonic resolution in relatively large volumes of biological tissue [4].

The acoustic waves from the initial sources reach the surface of the tissue with various time delays and can be measured by ultrasound receivers or by optical methods. The exited OA signal is locally determined by the electromagnetic absorption and scattering properties, including the thermal diffusivity and thermal expansion coefficient as well as the elastic properties of the sample [5]. The characteristics of the outgoing acoustic wave can be used to determine the initial acoustic source spatial distribution by applying suitable algorithms.

One way of detecting the surface waves is by scanning transducers, i.e. a focused ultrasound transducer scans along the sample surface. However, by doing so images cannot be acquired in real time. Alternatively, transducer arrays of piezoelectric materials [6,7] can be used. They enable numerically efficient and stable algorithms for thermoacoustic tomography. With such a system Khokhlova et al. [8] were able to record a piece of liver with a diameter of $3 \mathrm{~mm}$ located at a depth of $2 \mathrm{~cm}$ in diluted milk. Manohar et al. introduced the first Photoacoustic Mammoscope, applying parallel plate geometry where the breast is gently compressed between a glass plate and a flat ultrasound detector matrix [9]. However, despite of the progresses made, piezoelectric detection systems still suffer from limited resolution and speed regardless of their high sensitivity.

Other attractive methods to detect the pressure wave at the surface are optical methods having a high resolution at a slight cost of sensitivity. One of the main advantages of all optical systems is that no contact with the surface is necessary. This makes OA imaging particularly non-invasive, which is a great advantage under clinical conditions. For instance, single Schlieren images can be applied to reconstruct pressure sources inside a sample, enabling real-time imaging of absorbing structures inside tissue [10]. Another approach is to measure the pressure dependent reflection of a collimated probe beam at the refractive index mismatch of the detection plane at different time delays [11]. The 
resulting 2D snapshot of the pressure distribution is then used to reconstruct a 3D image with a depth resolution of 15 microns and a lateral resolution of $\sim 100$ microns. Meyer et al. tested an OA system with holographic diffractive steering [12] allowing for the generation ultrasonic fields with both temporal and spatial control. The surface displacement can also be imaged using interferometric measurements. Carp et al. presented a technique termed POIsi (pulsed optoacoustic interferometric spectroscopic imaging) [13]. The system provided an intrinsic lateral resolution below 200 microns. Payne et al. presented another OA setup able to detect contour images of an artificial vessel with a diameter of $1 \mathrm{~mm}$ at a depth of $1.36 \mathrm{~cm}$ in an intralipid solution [14].

In this study, acoustic surface waves, generated in water by a $12 \mathrm{~ns}$ laser pulse $(\lambda=1064 \mathrm{~nm})$ were recorded with a laser Doppler vibrometer (LDV), a heterodyne interferometer, which is an excellent tool to study minute vibrations on a surface. The resulting dielectric breakdowns were imaged using a double shutter camera. The aim of the presented work is twofold. First of all, the feasibility of the all optical setup based on an LDV to record minute surface waves was tested. Secondly, the possibility to use the temporal evolution of the surface waves in determining the size and position of the OA source was studied. The size and position of the breakdown event determined from the surface wave measurements were compared with images from the double shutter camera.

\section{Measurement SETUP ANd instrumentation}

The working principle of the setup was based on a single laser pulse that had a high enough irradiance to surpass the dielectric breakdown threshold in the fluid. The dielectric breakdown leads to a plasma formation emitting a multispectral flash of light. The event also creates a rapidly expanding gas bubble and an associated strong pressure wave, a shock wave, emanating from the breakdown volume [15]. When reaching the free surface, the pressure wave creates surface waves on the free water surface. The spatial and temporal shape of the surface waves contains information on the location and shape of the dielectric breakdown volume. These were calculated from the LDV data, measuring the surface normal velocity of the water surface.

The measurements were performed using the setup shown in figure 1. The setup comprises a custom made rectangular glass tank with dimensions of $0.25 \times 0.25 \times 0.25 \mathrm{~m}$ filled with a volume of deionized water $\left(\sim 0.007 \mathrm{~m}^{3}\right)$, a pulsed $\mathrm{Nd}$ :YAG laser generating the optoacoustic source, a double shutter camera to photograph the dielectric breakdown and a LDV to measure the vertical velocity of the free water surface. 


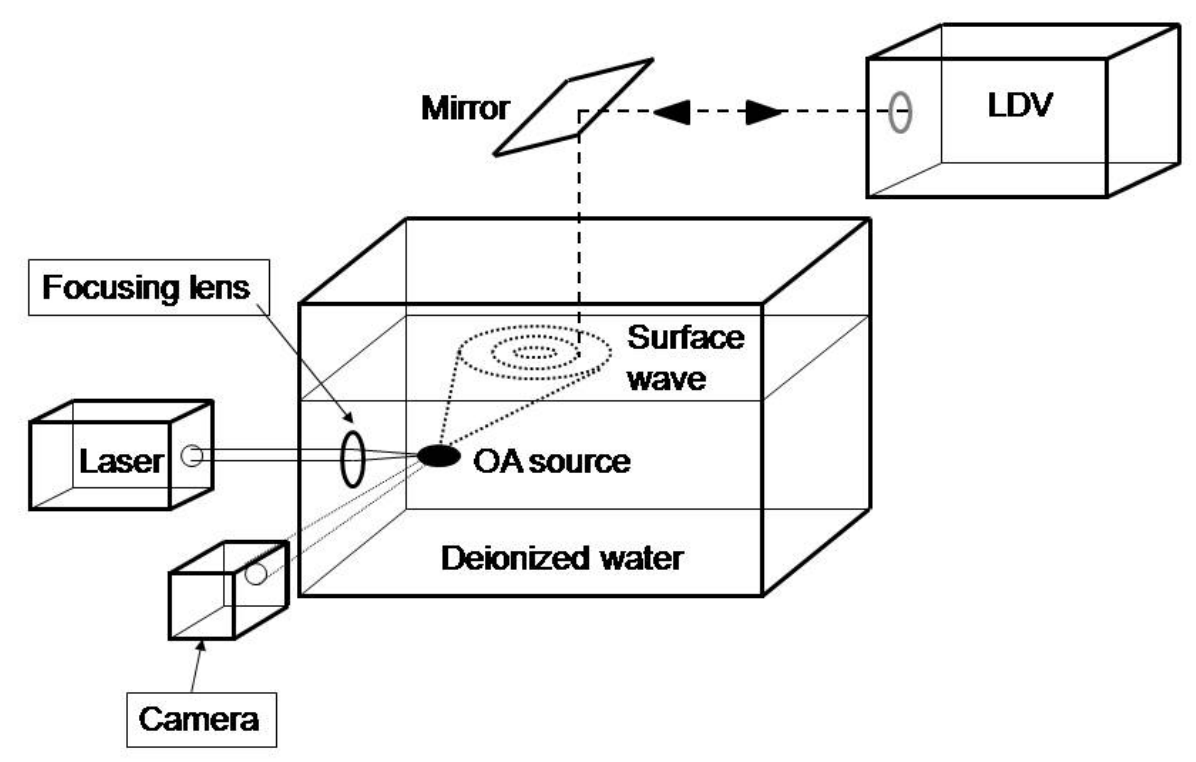

Fig. 1. The measurement setup. A single focused laser pulse from a $\mathrm{Nd:YAG} \mathrm{laser} \mathrm{entered} \mathrm{the}$ left side of the tank. The LDVs point of measurement was located at the free water surface. The point of measurement was positioned at a known horizontal offset to the focal point of the Nd:YAG laser beam. The event was photographed by a double shutter camera.

The Nd:YAG laser, a twin oscillator Spectron SL804T Q-switched laser from Spectron Lasers Ltd., UK, was operating in a TEM 00 mode with a near Gaussian beam intensity profile. The pulse energy was chosen to keep the irradiance at the focal volume well above the breakdown threshold for water [16]. The pulse length was $12 \mathrm{~ns}$ and the wavelength $\lambda=1064 \mathrm{~nm}$. The pulse energy was kept constant throughout the experiments and the energy in each pulse was recorded using a throughtransmission pulse energy meter. The laser pulse entered the tank horizontally at a known position relative to the sides and bottom of the tank and was focused inside the water volume using a focusing lens placed outside of the tank wall. The water temperature was measured throughout the experiments using a calibrated digital thermometer.

The LDV measurement beam entered the tank from above and was focused at the water surface. The vibrometer (Polytec PSV-300 from Polytec GmbH, Germany) has surface scanning capabilities that were not used in this case since only a specular reflection from the water surface could be detected. The LDV signal had a $2.5 \mathrm{MHz}$ sampling rate and a twelve-bit resolution. The LDV measurement point was placed at a horizontal offset to the focal point in order to reduce the influence from reflected surface waves on the measured signal. The laser induced breakdown events were recorded by a double shutter camera (PCO Sensicam double shutter, made by PCO AG, Germany). The camera was placed in front of the tank, in the same horizontal plane as the focal point. The pulsed laser, the double shutter camera, and the LDV were all triggered by the same signal. At each triggered event the data from the LDV and the double shutter camera was collected and stored digitally for off-line analysis. 


\section{RESULTS}

An example of the surface velocity data collected by the LDV is shown in figure 2a. From the data, the arrival times of the sound pulse and its echoes were determined. The different arrival times were matched to the different paths, as shown in figure 2. From this matching procedure both the acoustic pulse average speed of sound and the length of the dielectric breakdown volume was calculated. Finally, the calculated result on dielectric breakdown volume length was compared to the corresponding image taken by the double shutter camera.

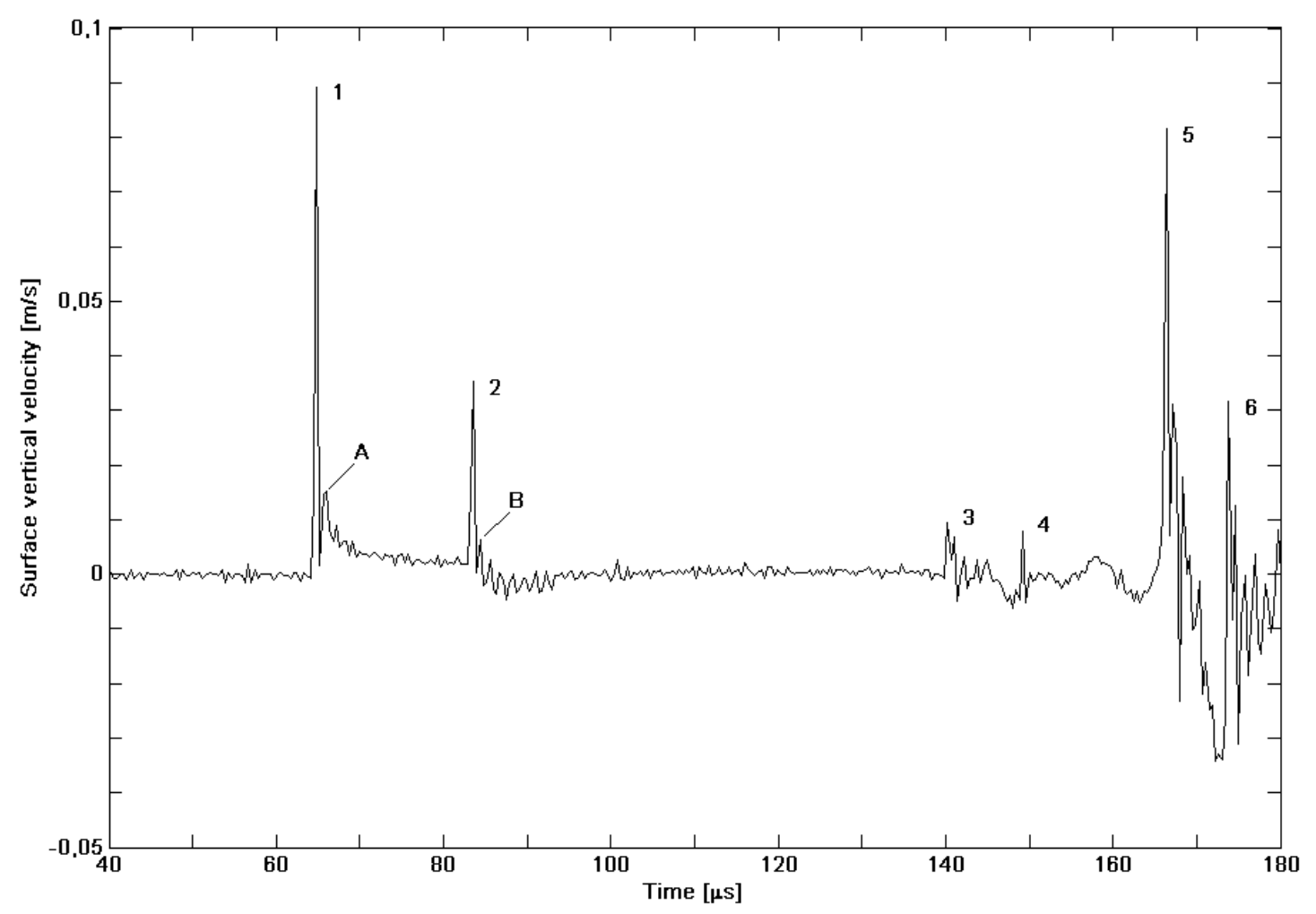

Fig. 2a. An example of a $L D V$ recording on the vertical velocity of the free surface. Eight different features from the recorded wave envelope were identified to originate from the optoacoustic source. The features 1 to 6 were identified to correspond to different paths taken by the acoustic wave, see figure $2 b$. In addition, the secondary peaks $A$ and $B$ adjacent to peaks 1 and 2 are believed to originate from the $O A$ source. Further investigations are needed to explain the mechanisms generating them. 


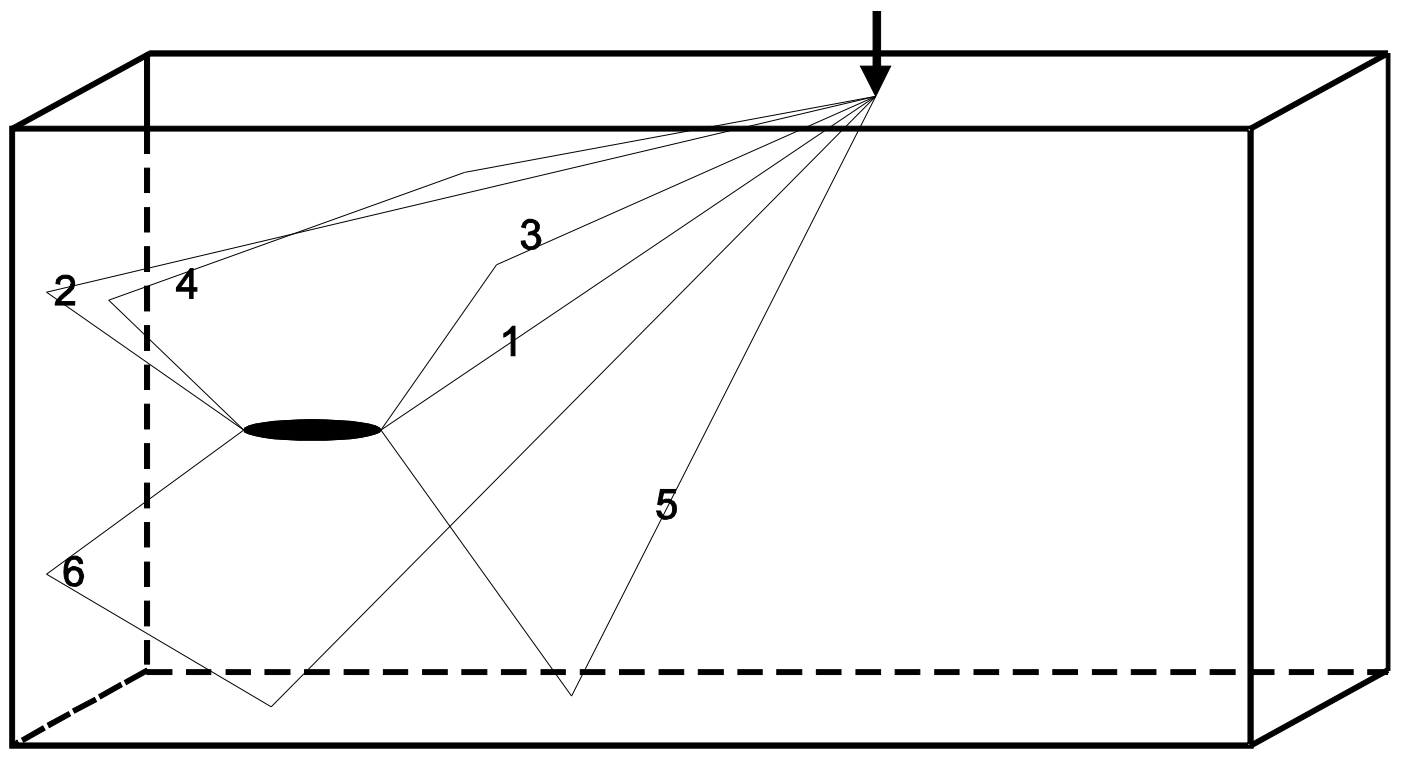

Fig $2 \boldsymbol{b}$. The figure shows the identified wave propagation paths. The numbers adjacent to each path correspond to the numbered features in figure 2a). The black ellipsoid in the figure marks the optoacoustic source and the arrow indicates the LDV measurement point. Note that paths 1, 3 and 5 start from the right end of the optoacoustic source and routes 2, 4 and 6 from the left.

\subsection{Speed of sound}

In the experiments, the water temperature varied between $19.5^{\circ} \mathrm{C}$ and $20.5^{\circ} \mathrm{C}$. Tabulated values for the linear speed of sound in water are given by [17]. From figure 3 it can be seen that the calculated sound speed are on average slightly higher than the tabulated velocities for the corresponding temperatures. 


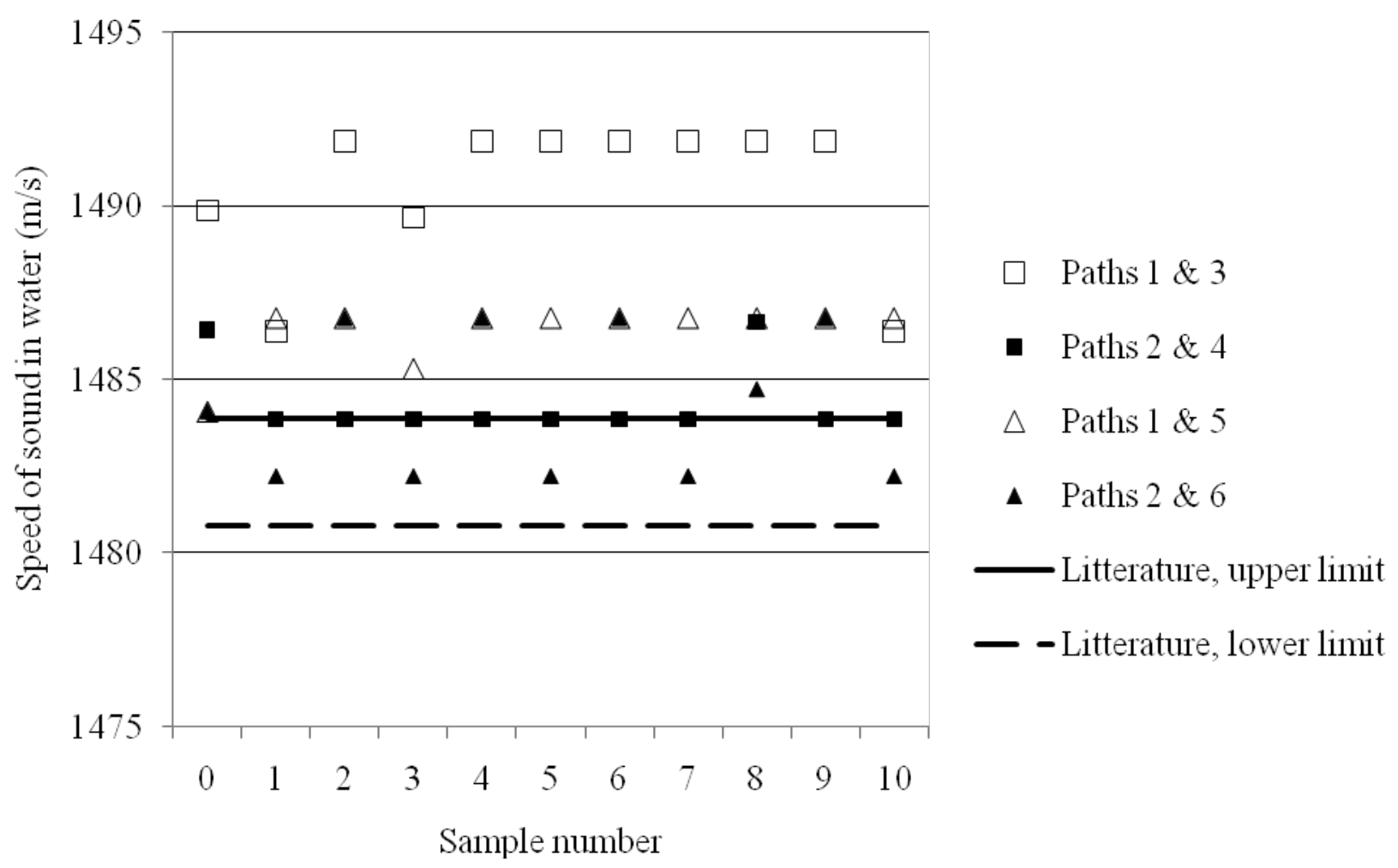

Fig. 3. Speed of sound in water. The markers indicate calculated average speed of sound for each dielectric breakdown event. The event numbers are shown on the x-axis. The horizontal lines and are the linear sound speed upper and lower limits at water temperatures $20^{\circ} \mathrm{C} \pm 0.5^{\circ} \mathrm{C}$, from [17].

The estimated inaccuracy of all distance measurements was $1 \mathrm{~mm}$, based on the $0.5 \mathrm{~mm}$ resolution of the measurement tools that were used. The inaccuracy of the calculated speed of sound depends on inaccuracy of the investigated path length. Using paths 1 and 3 an inaccuracy of $\delta c_{\text {mean }} \pm 13 \mathrm{~m} / \mathrm{s}$ is obtained, using path 2 and $4 \delta c_{\text {mean }} \pm 14 \mathrm{~m} / \mathrm{s}, 1$ and 5 gives $\delta c_{\text {mean }} \pm 10 \mathrm{~m} / \mathrm{s}$ and paths 2 and 6 gives $\delta$ $\mathrm{c}_{\text {mean }} \pm 11 \mathrm{~m} / \mathrm{s}$.

\subsection{Length of acoustic source}

In this study the origin of the acoustic wave, the optoacoustic source, was assumed to have the shape of a straight, slender cylinder that is placed along the axis of the Nd:YAG laser beam. The end positions of the imaginary cylinder were assumed to be unknown. By doing so, the matching procedure determined the positions of the ends of the cylinder. Paths 1, 3 and 5 were assumed to start from the right end of the cylinder and paths 2, 4 and 6 from the left end of the cylinder. As known variables, the position of LDV measurement point on the water surface and the position of the Nd:YAG laser beam entrance point at the tank wall was used. The average wave speed and the point at the tank wall where the normal to the wave front was reflected were calculated in the matching process. 
Once the speed of sound in the media is known through the matching procedure described above the position and length of the sound source could be calculated. The ends of the imaginary cylinder were calculated from peaks 2, 4 and 6, left end, and from peak 1, 3 and 5, right end, see figure 2a.

Figure $4 \mathrm{a}$ is a composite image of photos of eleven different laser pulses. Pulse 0 was a reference pulse randomly picked from a set of similar pulses obtained using a $f=16 \mathrm{~mm}$ lens. The photos 1-10 were obtained using the same experimental setup but fitted with a $f=40 \mathrm{~mm}$ lens.

Figure 4a show the plasma at some stage after initiation. The composite picture is assembled using pictures from eleven individual dielectric breakdown events. Picture 0 was obtained using the $f=16$ $\mathrm{mm}$ focusing lens. The picture show that the plasma volume is droplet shaped. The pictures 1-10 were obtained using the $f=40 \mathrm{~mm}$ focusing lens. These pictures clearly show that the plasma is not uniformly distributed in space but rather shaped as a randomly distributed series of smaller plasma bubbles, so called hot-spots.

The shock wave associated with the dielectric breakdown event can be considered to be created at the same instant as the initiation of the plasma and cavitation bubble [15]. After the initiation, the cavitation bubble expands, the plasma cools off and the expansion slows down. At some time instant in the bubble-expansion process the photo is taken. The photo then depicts the plasma in an expanded stage and is not depicting the OA source, i.e. the origin of the shock wave. Thus, the recorded shock wave carries information on the spatial extension of the OA source, which is the origin of the plasma and cavitation bubble, and the photo shows the plasma in the cavitation bubble at some later stage of expansion. This difference in size is seen in figure $4 b$ and $4 c$ since the plasma length determined from the photos is in general larger than the length calculated from the LDV measurements. 


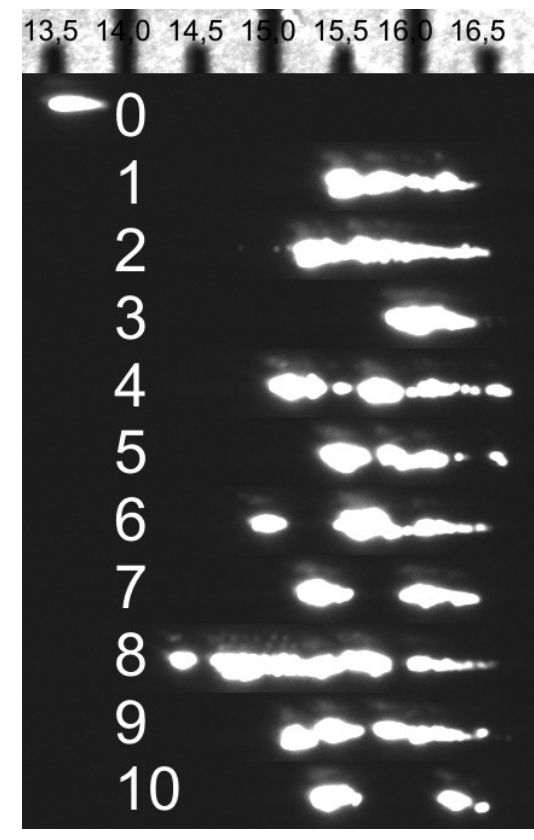

10

Place (mm)

Place (mm)

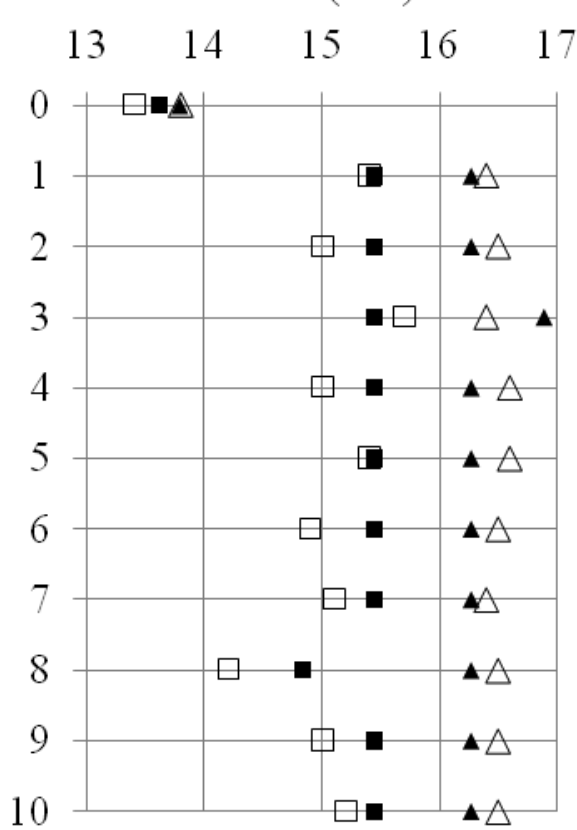

$\begin{array}{llllll}7 & 13 & 14 & 15 & 16 & 17\end{array}$

10

$\square$ StartPhoto

- Start1483 $\triangle$ StopPhoto

\ Stop 1483

\section{0 \\ 4 \\ 6 \\ 8 \\ 9 \\ 10}
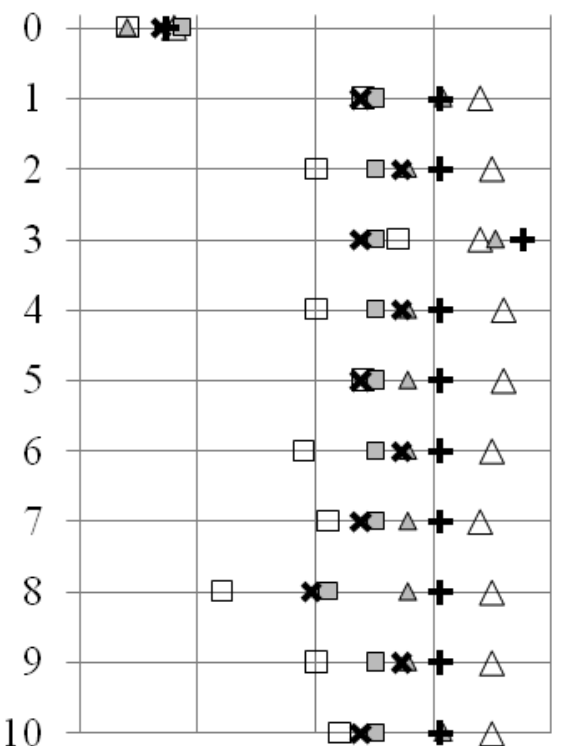

$\square$ StartPhoto

$\triangle$ StopPhoto

$\square$ Start2\&4

$\triangle$ Stop $1 \& 3$

X Start $2 \& 6$

Fig. 4. A comparison between individual dielectric breakdown volumes captured with the double shutter camera and right and left end positions determined from LDV data. In the a) picture the scale in the upper part of the composite image is in millimeters. In the b) figure unfilled markers indicate left end right ends determined from the photographs and black and gray markers indicate left and right ends calculated from the LDV signals. In the c) figure unfilled markers indicate left end right ends determined from the photographs and black markers indicate left and right ends calculated from the LDV signals assuming speed of sound is $1483 \mathrm{~m} / \mathrm{s}$. 


\subsection{Other observations}

The LDV recordings show secondary peaks located closely after the main peaks identified to originate from paths 1 and 2, see figure 5. The secondary peaks, marked as A and B in figure 5, appear at $\sim 0.8 \mu$ s later than the main velocity peak. The secondary peak is seen in both paths 1 and 2 and is therefore generated symmetrically at the OA source. It is also found that the event appears irrespective if the OA source is generated using the $f=16$ or the $f=40 \mathrm{~mm}$ focusing lens.
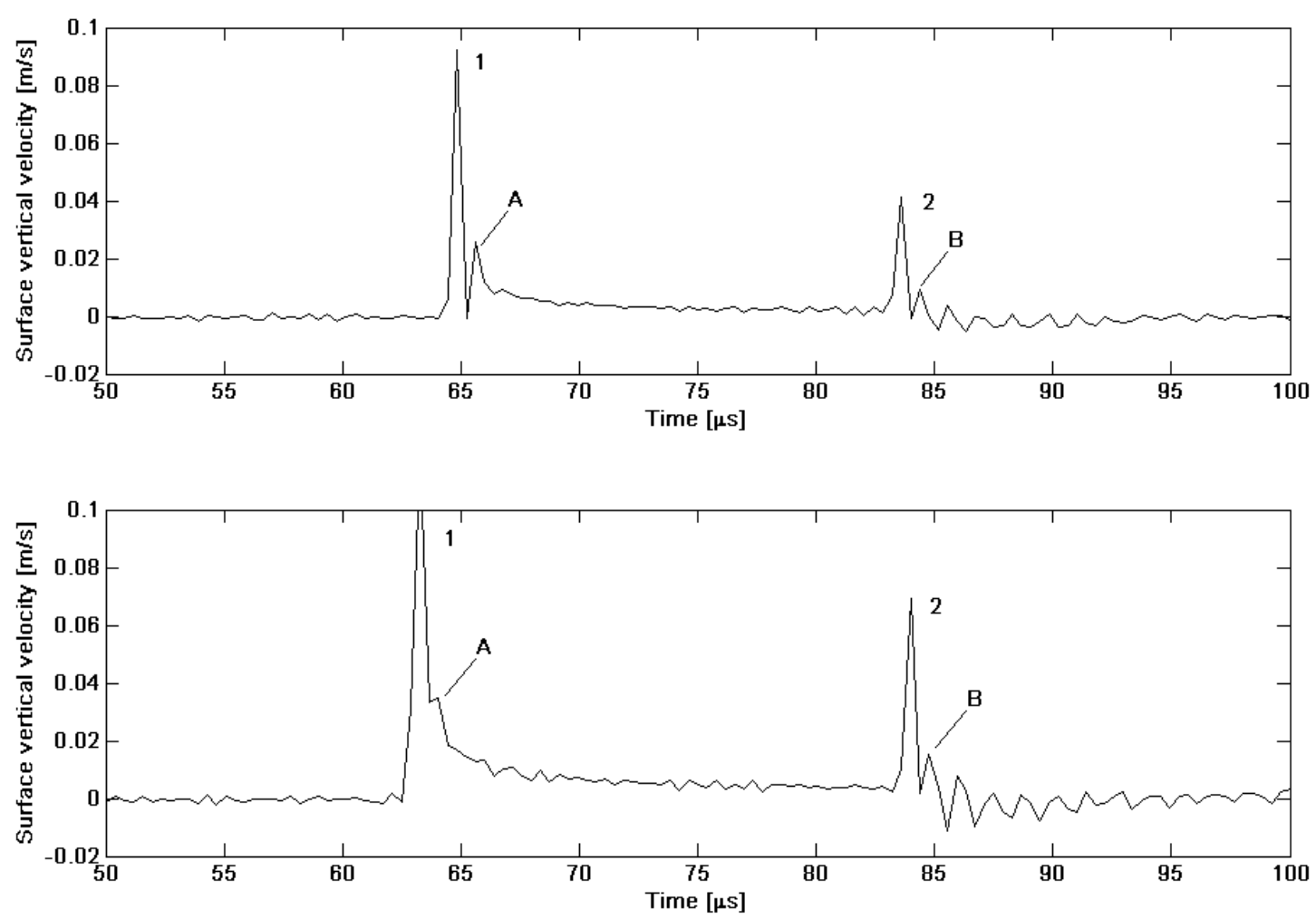

Fig. 5. LDV recordings from sample 0 (upper) and 1 (lower). The anomalous peaks are labeled $A$ and $B$. The sample numbers refer to the photos in figure 4.

At this preliminary stage, the secondary peak has been observed and found to belong to the OA source but its origin is not yet understood. Other investigators have reported similar results and the observed secondary peak could stem from the shock wave emanating from a repeated dielectric breakdown event, as in e.g. [18], or it could be generated from some other mechanism, like e.g. resonant relaxation as described by [19]. We conclude that in explaining the origin and properties of the secondary peak, the very initial stage of the OA source generation needs to be studied on nanosecond scale using methods other than the ones in the presented article.

\section{DISCUSSION}

This study shows that it is possible to use surface waves measured at a single point in detecting the size of an OA source embedded in water. The LDV, detecting the vertical velocity of the surface 
waves, is well suited for the task since it is a non-intrusive and rapid technique. When calculating the size of the OA source different echoes from the initiation of the embedded OA source was used. This straightforward and uncomplicated method yielded good estimates on the position and one of the dimensions, the length, of the embedded OA source. The limits on uncertainty in the method are traced back to the precision of the measurements of the linear dimensions as well as the temporal resolution of the LDV.

The method based on LDV detection of surface waves could be expanded from measuring at a point to measure along a line or at an area on the surface. The method expanded to a 1-D or a 2-D surface scan would eliminate the need for reflections at the boundaries, as in the method presented here, as well as it would result in a better estimate of the position and volumetric extent of the initial OA source.

It is well known that the pressure waves originating from a dielectric breakdown event initially can have wave velocities much larger than the normal speed of sound in water, see e.g. [15]. This is due to the increase in wave speed close to the volume of dielectric breakdown since the pressure at that point is high enough to induce a nonlinear behavior of the pressure wave, resulting in supersonic wave speeds. However, due to geometric attenuation and energy dissipation the supersonic wave will within a few hundred $\mu \mathrm{m}$ slow down to normal wave speed [15]. Although the distance and time of action for the supersonic wave is small compared to the total distance and travel-time, it influences the wave speed calculated from the experimental data. Although the inaccuracy of the method is larger than the spread in the velocity data the trend in the experimental results indicate that the speed of sound determined from the matching process is slightly larger than the normal speed of sound. The reason for this effect is believed to be the nonlinear wave propagation present in the first instant after the dielectric breakdown.

The technique presented in this study can be used for other transparent fluids and elastic solids. If it is assumed that the OA source can be of weaker nature, i.e. a less efficient process than dielectric breakdown, then the technique could potentially be used in particulate suspensions, tissue and other optical scattering materials.

The observation of a secondary peak appearing a short instant after the primary surface velocity peak is interesting since it implies that the surface wave velocity is suddenly increased. This observation is yet unexplained and calls for further investigations. Since the event stems from the initial instant of the dielectric breakdown the OA source further studies has to be made on a nanosecond scale using other techniques than used here.

\section{CONCLUSION}

Our results show that a point measuring LDV is well suited to determine the position and size, in one dimension, of an OA source embedded in water. The technique also gives estimates on the average speed of sound in the medium. The size of the OA source determined form the LDV measurements matched very well the length of the associated dielectric breakdown volume determined from photographs. The uncertainty in the determined average speed of sound was about $1 \%$ and the uncertainty in OA source position and length was about $1 \mathrm{~mm}$. 
The observation of secondary peak close to the primary surface velocity peak calls for further examinations of the initial instant of the OA source generation.

The all optic LDV based technique applied in this study is quite general and can be used as a point measuring technique or to be expanded to a line- or surface scanning technique extracting more of the information the surface wave carries on the location, shape and other properties of the embedded optoacoustic source. It is also concluded that the technique can potentially be used if the OA source is generated from a weaker mechanism than a dielectric breakdown. 


\section{REFERENCES}

1. P. K. Kennedy, D. X. Hammer and B. A. Rockwell, Quantum Electronics 21, 155 (1997).

2. A. Vogel, R. Engelhardt, U. Behnle and U. Parlitz, Applied Physics B: Lasers and Optics 62, 173 (1996).

3. A. Vogel, P. Schweiger, A. Frieser, M. Asiyo and R. Birngruber, IEEE Journal of Quantum Electronics 21, 2240, (1990)

4. A. A. Aliverdiev, A. A. Amirova and M. G. Karimov, Quantum Electronics 30, 1115 (2000).

5. M. H. Xu and L.H.V. Wang, Review of Scientific Instruments 77, 041101-1 (2006).

6. A. A. Karabutov, N. B. Podymova and V. S. Letokhov, Applied Physics B-Lasers and Optics 63, 545 (1996).

7. T. D. Khokhlova, I. M. Pelivanov, V. V. Kozhushko, A. N. Zharinov, V. S. Solomatin and A. A. Karabutov, Applied Optics 46, 262 (2007).

8. P. Burgholzer, C. Hofer, G. Paltauf, M. Haltmeier and O. Scherzer, IEEE Transactions on Ultrasonics Ferroelectrics and Frequency Control 52, 1577 (2005).

9. S. Manohar, A. Kharine, J. C. G. van Hespen, W. Steenbergen and T. G. van Leeuwen. Phys. Med. Biol. 50, 2543 (2005).

10. J. J. Niederhauser, D. Frauchiger, H. P. Weber and M. Frenz, Applied Physics Letters 81, $571(2002)$.

11. K. P. Kostli, D. Frauchiger, J. J. Niederhauser, G. Paltauf, H. P. Weber and M. Frenz, IEEE Journal of Selected Topics in Quantum Electronics 7, 918 (2001).

12. A. Meyer, S. Gspan, S. Bernet and M. Ritsch-Marte, Journal of Applied Physics 96, 5886 (2004).

13. S. A. Carp, A. Guerra, S. Q. Duque and V. Venugopalan, Applied Physics Letters 85, 5772 (2004).

14. B. P. Payne, V. Venugopalan, B. B. Mikic and N. S. Nishioka, Journal of Biomedical Optics 8,273 (2003).

15. A. Vogel, S. Busch, and U. Parlitz, Journal of the Acoustical Society of America 100, 148 (1996)

16. J. Niemi, T. Löfqvist and P. Gren, in: SPIE Proceedings 7022-9, Advanced Laser Technologies 3.-7. 9. 2007, Levi Finland,

17. N. Bilaniuk and G. S. K. Wong, Journal of the Acoustical Society of America 93, 1609 (1993).

18. I. Akhatov, O. Lindau, A. Topolnikov, R. Mettin, N. Vakhitova and W. Lauterborn, Physics of Fluids 13, 2805 (2001).

19. A. A. Karabutov, G. A. Larichev, I. M. Maksimov, and N. B. Podymova, Acoustical Physics $52,582(2006)$. 